\title{
Comparison of Male and Female Prolactinoma Patients Requiring Surgical Intervention
}

\author{
Frederick Yoo ${ }^{1}$ Carmen Chan ${ }^{1} \quad$ Edward C. Kuan ${ }^{1}$ Marvin Bergsneider ${ }^{2}$ Marilene B. Wang ${ }^{1}$
} ${ }^{1}$ Department of Head and Neck Surgery, David Geffen School of
Medicine at UCLA, Los Angeles, California, United States
2 Department of Neurosurgery, David Geffen School of Medicine at
UCLA, Los Angeles, California, United States

\begin{abstract}
Address for correspondence Marilene B. Wang, MD, Department of Head and Neck Surgery, UCLA David Geffen School of Medicine, 10833 Le Conte Avenue, 62-132 CHS, Los Angeles, CA 90095-1624, United States (e-mail: mbwang@ucla.edu).
\end{abstract}

J Neurol Surg B 2018;79:394-400.

\begin{abstract}
Objectives Prolactinomas are the most common functional pituitary adenoma. Symptoms of a prolactinoma stem from hormonal causes (menstrual irregularities, galactorrhea, and reduced libido) or from tumor mass effect (visual changes and headache). Gender differences have been noted in prolactinomas, with males presenting with larger tumors and sequelae of mass effect, while females present commonly with hormonal symptoms. The purpose of this study is to evaluate differences in patient and disease characteristics, and outcomes between male and female prolactinoma patients undergoing surgery.

Design This was a retrospective chart review.

Setting This was done at the tertiary medical center.

Participants The medical records of prolactinomas patients who underwent endoscopic endonasal surgery between March 2008 and August 2016 were reviewed.

Main Outcome Measures Demographic information, tumor characteristics, and treatment characteristics and outcomes were collected. Statistical analysis was performed using chi-squared test or Student's t-test as applicable.

Results Seventy-nine patients were identified, 22 males and 57 females. The average age for males was 38 years and for females was 35 years. Males were more likely to present with decreased libido $(p<0.0001)$, whereas females more often presented with galactorrhea $(p<0.0001)$ and menstrual irregularities. Tumor size was larger in males $(p=0.0044)$ with higher likelihood of suprasellar extension $(p=0.0409)$ and cavernous sinus invasion

\section{Keywords}

- pituitary adenomas

- prolactinoma

- gender

- endoscopic $(p=0.0026)$. Males were more likely to have a subtotal resection rather than gross total resection ( $p=0.0086)$ and less likely to have normalization of prolactin levels following surgery $(p=0.0019)$

Conclusion Male prolactinoma patients tend to have larger tumors with more aggressive features. This may have a role in the differences in outcomes noted in this study.
\end{abstract}

\section{Introduction}

Prolactinomas are the most common of all pituitary adenomas, accounting for 51 to $66 \%$ of these tumors. ${ }^{1}$ Common presenting symptoms include headache, visual impairment, galactorrhea, and effects of hypogonadism including ame-

received

July 16,2017

accepted after revision

November 19, 2017

published online

December 26, 2017

norrhea, infertility, and erectile dysfunction. ${ }^{2}$ Dopamineagonist therapy including cabergoline and bromocriptine are the first-line treatments for prolactinomas. However, some patients will require surgical treatment because they are refractory to medical therapy, are intolerant to medication side effects, desire pregnancy, or due to patient

(c) 2018 Georg Thieme Verlag KG Stuttgart · New York
DOI https://doi.org/ 10.1055/s-0037-1615748. ISSN 2193-6331. 
preference. ${ }^{3}$ The endoscopic endonasal approach has largely become the standard surgical approach due to magnified and panoramic views, decreased invasiveness, and high rate of successful outcomes. ${ }^{4-6}$ Previous analyses have reported that up to $93 \%$ patients with prolactinomas achieve remission with surgery, with variability due to the extent of invasion and size of the tumor. ${ }^{1,7-9}$

Studies have noted some gender differences in prolactinomas. Hyperprolactinemia has been shown to be more common among women with the incidence varying with age. ${ }^{10}$ However, prolactin levels have been found to be higher in men, which has been attributed to be likely due to the increased frequency of macroadenomas at presentation. ${ }^{11}$ These findings have not been completely elucidated. Some attribute it to the later onset of vague symptoms resulting in delayed diagnosis, while others to the tumor biology. ${ }^{12-15}$ Men typically present with decreased libido and impotence, whereas women tend to have more clear-cut symptoms including menstrual dysfunction and galactorrhea. ${ }^{15,16}$ Thus far, there have also been conflicting results determining if there is a difference in response to medical treatment in males and females. ${ }^{15,17}$

Although there are studies looking at these gender differences in prolactinomas, there is limited data evaluating these two cohorts in the setting of surgical management. One recent study evaluating 142 surgical cases of prolactinoma showed that gender was an independent factor in predicting persistent disease following surgery. ${ }^{7}$ The aim of this study is to compare males and females with prolactinomas that had undergone transnasal transsphenoidal endoscopic surgery for management of their prolactinomas at a single institution, comparing their characteristics at presentation, response to medical therapy, and surgical outcomes.

\section{Methods}

Retrospective review was performed on all patients who underwent transnasal transsphenoidal endoscopic resection of prolactinoma from March 2008 to June 2016. Data were extracted from patient charts including demographics, type of medical therapy, length of medical therapy trial, response to medical therapy, reasons for proceeding to surgery, presenting symptoms, presenting prolactin level, tumor size at surgery, presence of suprasellar extension, presence of cavernous sinus invasion, type of resection achieved during surgery, presence of intraoperative cerebrospinal fluid (CSF) leak, complications, postoperative prolactin level at 2 months, length of stay, postoperative radiation therapy, and continued medical therapy following surgery. Statistical analysis was performed using Fisher's exact test or chi-square test for categorical variables, and two-tailed $t$-tests for continuous variables. Assumption of equal or unequal variance was determined by performing the $F$-test of the equality of variances and performed as indicated. Significance was set at a $p$-value of less than 0.05 .

\section{Results}

In total, there were 79 patients who underwent surgical treatment for prolactinoma included in this study. Twenty- two patients were male and 57 patients were female. Preoperative patient characteristics are shown in - Table 1. Average age at the time of surgery was 38 years for males and 35 years for females $(p=0.2932)$. Males tend to have higher frequency of atypical adenoma, but this was not statistically significant $(p=0.0750)$. Presenting symptoms differed between males and females. Males tend to present with more decreased libido ( $p<0.0001$ ) and females tend to present more often with galactorrhea $(p<0.0001)$. The most common presenting symptom for males was decreased libido (50\%) and the most common presenting symptom for females was menstrual irregularities (77.2\%). Males were found to have higher average prolactin levels at diagnosis at $2,281 \mathrm{ng} / \mathrm{mL}$ compared with females with $532.9 \mathrm{ng} / \mathrm{mL}$, but this was not statistically significant. Males were found to have significantly larger tumor size at surgery $(p=0.0044)$, higher rate of suprasellar extension ( $p=0.0409$ ), and higher prevalence of cavernous sinus invasion compared with females $(p=0.0026)$. The distribution of tumors into categories of micro-, macro-, and giant adenomas was also significant $(p=0.0062)$, with note that there were no giant adenomas in the female group. The $\mathrm{K}_{\mathrm{i}}-67$ labeling index on final pathology reports was slightly higher on average for males than females, 4.77 and 3.52, respectively, but this was not statistically significant.

The summary of the medical therapy received by the patient population is presented in - Table 2. No significant differences were noted between males and females regarding percentage of patients trialed on medical therapy with a dopamine agonist prior to surgery, prolactin level response to medical therapy, tumor size response to medical therapy, and length of medical therapy. For males, the most common reasons for pursuing surgery were partial/no response to dopamine-agonist therapy and persistent/worsening symptoms. For females, the most common reasons for pursuing surgery were partial or no response to dopamine-agonist therapy followed by intolerance of side effects of medical therapy.

The operative outcomes are presented in - Table 3. Female patients were found to have significantly higher rates of gross total resection $(p=0.0086)$ and normalization of serum prolactin levels 2 months following surgery $(p=0.0016)$ compared with male patients. There was no significant difference between the two genders in regard to complications from surgery, presence of an intraoperative CSF leak, length of stay, or undergoing postoperative radiotherapy. Male patients tend to require continued medical therapy with dopamine-agonist therapy following surgery compared with female patients, and this difference approached significance ( $p=0.0536)$.

Subgroup analysis of the gender groups with respect to size of tumor in categories of micro-, macro-, and giant adenomas was performed ( - Table 4 ) showing that in males, there was a statistically significant increase in prolactin level with size (Spearman rho $=0.6184, p=0.0022$ ) and a near significant increase in $\mathrm{K}_{\mathrm{i}}$-67 labeling index with size (Spearman rho $=0.4021, p=0.0636$ ). In females, however, there were no significant increases in prolactin level with size (Spearman rho $=0.2267, p=0.0899$ ) and $\mathrm{K}_{\mathrm{i}}-67$ labeling 
Table 1 Preoperative patient and tumor characteristics

\begin{tabular}{|c|c|c|c|}
\hline & Males $(n=22)$ & Females $(n=57)$ & $p$-Value \\
\hline Age at surgery $(\mathrm{y})$ & 38 (SD 15.56) & 35 (SD 9.28) & 0.2932 \\
\hline \multicolumn{4}{|l|}{ Hormone secretion } \\
\hline PRL only & 19 & 46 & \multirow[t]{2}{*}{0.7461} \\
\hline PRL + other & 3 & 11 & \\
\hline \multicolumn{4}{|l|}{ Pituitary adenoma grade } \\
\hline Typical adenoma & 20 & 57 & \multirow[t]{2}{*}{0.0750} \\
\hline Atypical adenoma & 2 & 0 & \\
\hline \multicolumn{4}{|l|}{ Presenting symptoms } \\
\hline Decreased libido & $11(50 \%)$ & $3(5.3 \%)$ & \multirow[t]{3}{*}{$<0.0001$} \\
\hline Gynecomastia & $9(40.9 \%)$ & $\mathrm{N} / \mathrm{A}$ & \\
\hline Menstrual irregularities & $\mathrm{N} / \mathrm{A}$ & $44(77.2 \%)$ & \\
\hline Galactorrhea & $2(9.1 \%)$ & $35(61.4 \%)$ & $<0.0001$ \\
\hline Headache & $9(40.9 \%)$ & $17(29.8 \%)$ & 0.4254 \\
\hline Visual disturbance & $6(27.3 \%)$ & $7(12.3 \%)$ & 0.1723 \\
\hline PRL level at diagnosis (ng/mL) & $2,281.0$ (SD 4,715.82) & 532.9 (SD 1,213.87) & 0.1000 \\
\hline Tumor size at surgery $(\mathrm{mm})$ & 18.9 (SD 11.73) & 10.7 (SD 5.37) & 0.0044 \\
\hline \multicolumn{4}{|l|}{ Tumor size category } \\
\hline Micro & 7 & 34 & \multirow[t]{3}{*}{0.0062} \\
\hline Macro & 12 & 23 & \\
\hline Giant & 3 & 0 & \\
\hline \multicolumn{4}{|l|}{ Suprasellar extension } \\
\hline Yes & 9 & 10 & \multirow[t]{2}{*}{0.0409} \\
\hline No & 13 & 47 & \\
\hline \multicolumn{4}{|l|}{ Cavernous sinus invasion } \\
\hline None & 13 & 52 & \multirow[t]{3}{*}{0.0026} \\
\hline Unilateral & 8 & 5 & \\
\hline Bilateral & 1 & 0 & \\
\hline $\mathrm{K}_{\mathrm{i}}-67$ labeling index (average) & 4.77 & 3.52 & 0.4240 \\
\hline
\end{tabular}

Abbreviations: N/A, not available; PRL, prolactin; SD, standard deviation.

index with size (Spearman rho $=0.1164, p=0.3886$ ). In both genders, the rate of subtotal resection increased significantly with tumor size $(p=0.0066$ for males and $p=0.0224$ for females), and differences in rates of prolactin normalization after surgery with tumor size were only significant in males ( $p=0.0035)$. Subgroup analysis of males presenting with hormone-related symptoms was performed (-Table 5) and showed that males who present with gynecomastia, galactorrhea, or decreased libido had smaller tumor sizes, though this was only statistically significant for galactorrhea $(p=0.0030)$. In addition, patients who present with galactorrhea appeared to have statistically significant lower prolactin levels at diagnosis and $\mathrm{K}_{\mathrm{i}}-67$ indices ( $p=0.0449$ and $p=0.0158$, respectively). Patients with gynecomastia also appeared to have lower prolactin levels at diagnosis and lower $\mathrm{K}_{\mathrm{i}}-67$ indices, but this was not statistically significant.

\section{Discussion}

Gender differences in prolactinomas have been documented in previous studies; however, the reason for these differences has not yet been explained. Prolactinomas are more common in females, and this was confirmed in our series with more than twofold number of female patients compared with male patients in the time period of study. ${ }^{4}$ Moreover, in other series, females tend to be 10 years younger at the age of diagnosis, though this was not evident in our series with the age at the time of surgery being only slightly older in males. ${ }^{4,18}$ One thought is that the difference in incidence among the genders is related to differences in clinical presentation. As stated previously, the clinical manifestation of hyperprolactinemia in men and women differ in that men tend to have more insidious, and often clinically underestimated, symptoms of low libido and erectile dysfunction, 
Table 2 Summary of medical treatment prior to surgical intervention

\begin{tabular}{|c|c|c|c|}
\hline & Males $(n=22)$ & Females $(n=57)$ & $p$-Value \\
\hline \multicolumn{4}{|l|}{ Medical treatment with DA } \\
\hline Yes & 20 & 52 & \multirow[t]{2}{*}{0.9643} \\
\hline No & 2 & 5 & \\
\hline \multicolumn{4}{|c|}{ Prolactin level response to medical therapy } \\
\hline No & 2 & 12 & \multirow[t]{3}{*}{0.4813} \\
\hline Partial & 11 & 24 & \\
\hline Complete & 7 & 14 & \\
\hline \multicolumn{4}{|l|}{ Tumor size reduction } \\
\hline No & 10 & 32 & \multirow[t]{2}{*}{0.3737} \\
\hline Yes & 10 & 20 & \\
\hline Length of medical therapy (mo) & 31.75 & 32.43 & 0.9499 \\
\hline \multicolumn{4}{|l|}{ Reasons for pursuing surgery (\%) } \\
\hline Side effects to DA & $7(31.8 \%)$ & $26(45.6 \%)$ & \\
\hline Partial/no response to DA & $9(40.9 \%)$ & $36(63.2 \%)$ & \\
\hline Persistent/worsening symptoms & $8(36.4 \%)$ & $15(26.3 \%)$ & \\
\hline Patient preference & $0(0 \%)$ & $2(3.5 \%)$ & \\
\hline Desire for pregnancy & $\mathrm{N} / \mathrm{A}$ & $8(14.0 \%)$ & \\
\hline
\end{tabular}

Abbreviations: DA, dopamine agonist; N/A, not available.

Table 3 Operative outcomes

\begin{tabular}{|c|c|c|c|}
\hline & $\begin{array}{l}\text { Males } \\
(n=22)\end{array}$ & $\begin{array}{l}\text { Females } \\
(n=57)\end{array}$ & $p$-Value \\
\hline \multicolumn{4}{|l|}{ Resection } \\
\hline Gross total & 15 & 53 & \multirow[t]{2}{*}{0.0086} \\
\hline Subtotal & 7 & 4 & \\
\hline \multicolumn{4}{|c|}{ Normalization of PRL 2 mo after surgery } \\
\hline Yes & 13 & 52 & \multirow[t]{2}{*}{0.0019} \\
\hline No & 9 & 5 & \\
\hline \multicolumn{4}{|l|}{ Complications } \\
\hline Yes & 20 & 51 & \multirow[t]{2}{*}{1.000} \\
\hline No & 2 & 6 & \\
\hline \multicolumn{4}{|c|}{ Intraoperative CSF leak } \\
\hline Yes & 5 & 9 & \multirow[t]{2}{*}{0.5177} \\
\hline No & 17 & 48 & \\
\hline Length of stay $(\mathrm{d})$ & 2.90 & 2.32 & 0.2885 \\
\hline \multicolumn{4}{|c|}{ Postoperative radiation } \\
\hline Yes & 2 & 2 & \multirow[t]{2}{*}{0.3087} \\
\hline No & 20 & 55 & \\
\hline \multicolumn{4}{|c|}{ Required medical therapy postoperative } \\
\hline Yes & 7 & 7 & \multirow[t]{2}{*}{0.0536} \\
\hline No & 15 & 50 & \\
\hline
\end{tabular}

Abbreviations: CSF, cerebrospinal fluid; PRL, prolactin. compared with females who present most often with menstrual cycle irregularities; thus males would present at a later age. ${ }^{18}$ Our study confirmed this tendency, as the most common presenting symptom for males and females was decreased libido and menstrual irregularities, respectively. Support for the hypothesis that females present more often due to more obvious clinical signs and symptoms as opposed to a true increased incidence is found in autopsy studies where the prevalence of prolactinomas was similar in males and females postmortem. ${ }^{19}$ Another hypothesis points to hormonal differences between males and females leading to the difference in gender incidence, with one study showing greater incidence in females during child-bearing age, which becomes more even, in the fifth to seventh decades of life between the two genders. ${ }^{10}$ In addition, there have been case reports of prolactinomas induced in male-to-female transsexual patients, possibly due to hormonal therapy, though causation cannot be definitively determined in these cases. $^{20-23}$

The analysis from this study also revealed that males had significantly larger tumors and thus more symptoms related to mass effect, such as visual disturbances, compared with females. In addition, men had significantly higher rates of cavernous sinus invasion and suprasellar extension, which are clinical markers of advanced and aggressive tumors. The difference in tumor size and presentation of males with visual disturbances has been well described in the literature. ${ }^{7,12,14,15,24}$ Most often, the differences in tumor size and aggressiveness are attributed to delays in detection in men; however, some findings have advocated for a difference in 
Table 4 Subgroup analysis of male and female groups based on tumor size

\begin{tabular}{|c|c|c|c|c|}
\hline \multicolumn{5}{|l|}{ Males } \\
\hline & Micro $(n=7)$ & Macro $(n=12)$ & Giant $(n=3)$ & $p$-Value \\
\hline Prolactin level at diagnosis (ng/mL) & 172.0 & 960.4 & $12,404.3$ & \\
\hline $\mathrm{K}_{\mathrm{i}}-67$ labeling index & 2.14 & 2.92 & 18.33 & \\
\hline Subtotal resection (\%) & $0(0 \%)$ & $4(33.3 \%)$ & $3(100 \%)$ & 0.0066 \\
\hline Prolactin normalization after surgery (\%) & $7(100 \%)$ & $6(50 \%)$ & $0(0 \%)$ & 0.0035 \\
\hline Correlation & \multicolumn{3}{|l|}{ Spearman rho } & p-Value \\
\hline Tumor size to prolactin level at diagnosis & \multicolumn{3}{|l|}{0.6184} & 0.0022 \\
\hline Tumor size to $\mathrm{K}_{\mathrm{i}}-67$ labeling index & \multicolumn{3}{|l|}{0.4021} & 0.0636 \\
\hline \multicolumn{5}{|l|}{ Females } \\
\hline & Micro $(n=34)$ & Macro $(n=23)$ & Giant $(n=0)$ & $p$-Value \\
\hline Prolactin level at diagnosis & 310.3 & 862.1 & $\mathrm{~N} / \mathrm{A}$ & \\
\hline $\mathrm{K}_{\mathrm{i}}-67$ labeling index & 3.44 & 3.65 & $\mathrm{~N} / \mathrm{A}$ & \\
\hline Subtotal resection & 0 & $4(17.4 \%)$ & $\mathrm{N} / \mathrm{A}$ & 0.0224 \\
\hline Prolactin normalization after surgery & $32(94.1 \%)$ & $20(87.0 \%)$ & $\mathrm{N} / \mathrm{A}$ & 0.3837 \\
\hline Correlation & \multicolumn{3}{|l|}{ Spearman rho } & $p$-Value \\
\hline Tumor size to prolactin level at diagnosis & \multicolumn{3}{|l|}{0.2267} & 0.0899 \\
\hline Tumor size to $\mathrm{K}_{\mathrm{i}}-67$ labeling index & \multicolumn{3}{|l|}{0.1164} & 0.3886 \\
\hline
\end{tabular}

Abbreviation: N/A, not available.

tumor biology to be the true cause of these differences. Studies have noted differences in tumor histopathological characteristics between the genders, with male tumors showing greater rates of cellular atypia and higher amounts of $\mathrm{K}_{\mathrm{i}}-67$ labeling index staining on histopathology. ${ }^{12,14,24,25}$ In our data, the male group did have more diagnoses of atypical adenoma and did show a slightly higher $\mathrm{K}_{\mathrm{i}}-67$ labeling index; however, these findings were not significant. Other studies have presented evidence that differential expression of estrogen receptor $\alpha$ and transforming growth factor $\beta 1$ may play a role in the difference seen in tumor pathogenesis between the genders, though the results of these studies are yet to be confirmed. ${ }^{13,26}$

Previous studies have also shown differences in disease history between men and women with prolactinomas. Specifically, men have been previously shown to have shorter duration of symptoms prior to surgery, higher levels of preoperative serum prolactin levels, and more medically refractory disease. ${ }^{12,14,15,24,27,28}$ Males in our patient population did have higher prolactin levels at diagnosis, but this

Table 5 Subgroup analysis of males with hormonal symptoms at presentation

\begin{tabular}{|l|l|l|l|l|l|}
\hline & BMI $\left(\mathrm{kg} / \mathrm{m}^{2}\right)$ & Tumor size $(\mathrm{mm})$ & PRL level at diagnosis & Subtotal resection & $\mathrm{K}_{\mathrm{i}}$-67 labeling index \\
\hline Gynecomastia \\
\hline No $(n=13)$ & 31.75 & 21.69 & $3,312.9$ & $6(46.1 \%)$ & 6.38 \\
\hline Yes $(n=9)$ & 33.65 & 14.89 & 790.4 & $1(11.1 \%)$ & 2.44 \\
\hline$p$-Value & 0.4811 & 0.1624 & 0.1599 & 0.1649 & 0.1241 \\
\hline Galactorrhea & \multicolumn{5}{|l|}{} \\
\hline No $(n=20)$ & 33.03 & 19.95 & $2,495.1$ & $7(35 \%)$ & 5.15 \\
\hline Yes $(n=2)$ & 27.44 & 8.50 & 140.0 & $0(0 \%)$ & 1.00 \\
\hline$p$-Value & 0.3879 & 0.0030 & 0.0449 & 0.5454 & 0.0158 \\
\hline Decreased libido & & & $4(36.4 \%)$ \\
\hline No $(n=11)$ & 33.79 & 21.91 & $2,337.9$ & $3(27.3 \%)$ & 5.36 \\
\hline Yes $(n=11)$ & 31.26 & 15.91 & $2,224.1$ & 1 & 4.18 \\
\hline$p$-Value & 0.3438 & 0.2399 & 0.9566 & 0.6940 \\
\hline
\end{tabular}

Abbreviations: BMI, body mass index; PRL, prolactin. 
was not statistically significant. There was no difference in length of medical therapy between the groups. Females in our study group did have a greater percentage of patients who had completely medically resistant tumors to dopamine-agonist therapy. When grouping together the nonresponsive and partially responsive tumors in each gender, the rates were very similar at $59 \%$ for males and $63 \%$ for females. This is in contrary to previous reports which have noted that dopamine-agonist-resistant prolactinomas tend to be larger in size, have increased angiogenesis, have more cellular atypia, have increased invasiveness, and are more likely in male patients. ${ }^{28}$

There were also notable outcomes differences between the two genders noted in our study. Males were found to have significantly lower rates of gross total resection, normalization of prolactin levels 2 months after surgery, and were approaching significance for requiring continued medical therapy with a dopamine agonist following surgery. These findings are in line with previous reports which have shown lower rates of remission in men than in women. ${ }^{7,13,27}$ It is likely that the rate of remission after surgery is related to the size and invasiveness of the tumors, as this would make intuitive sense. However, a previous study showed that cavernous sinus invasion was associated with lack of remission postoperatively in univariate analysis but not in multivariate analysis. ${ }^{7}$ In addition, a review of surgical removal of microprolactinomas showed high remission rates of 83 to $100 \%$ when performed by experienced surgeons, lending credence to size being a factor in outcomes. ${ }^{1}$

In our subgroup analyses, we found that the prolactin level at diagnosis is related to tumor size, an association which is already been described. ${ }^{9}$ There was a significant correlation between tumor size and $\mathrm{K}_{\mathrm{i}}-67$ labeling index in males but not in females. However, this is likely due to the fact that there were not any female patients with giant prolactinomas included in the study group, with the largest female tumor at $28 \mathrm{~mm}$ in size. As would be expected, our results showed that patients with microadenomas were significantly more likely to have gross total resections in both genders. Subgroup analysis of males with hormone-related symptoms (gynecomastia, galactorrhea, and decreased libido) revealed that these patients tend to present with smaller tumor sizes, lower levels of prolactin at diagnosis, and lower $\mathrm{K}_{\mathrm{i}}-67$ labeling indices, of which these were only statistically significant for patients presenting with galactorrhea. Of note, there was no statistically significant difference in body mass index between males with gynecomastia and males without gynecomastia, with averages of 33.68 and 31.75 , respectively $(p=0.4811)$. These findings may represent that male patients presenting with hormone-related symptoms may have biologically different tumors which behave more like female prolactinomas than males who present with compressive symptoms. However, due to limited number of patients in this analysis, further study is warranted to draw any conclusions.

As noted previously, delays in detection of prolactinomas in males compared with females are the most commonly cited reason for differences in clinical presentation and outcomes between the two genders. There have also been studies which point to tumor biology, through histopathological analyses and evidence of dopamine-agonist resistance, as the cause of these differences in presentation and outcomes. It is likely that the differences noted in the two genders are a combination of both theories. Current clinical practice guidelines set forth by the Endocrine Society recommend morning testosterone testing in males with signs and symptoms of hypogonadism, including low libido and erectile dysfunction and further testing of the pituitary axis only if this laboratory test should prove to be abnormal. ${ }^{29}$ However, normal testosterone levels may be seen in patients with mild hyperprolactinemia and in up to $30 \%$ of patients with prolactinomas. ${ }^{30}$ Due to this, clinicians should keep in mind the possibility of a prolactinoma in male patients who present with persistent symptoms of hypogonadism, even when previous testosterone testing has been within the normal range.

There are several limitations to our study. Our patient cohort were selected from patients who underwent surgery for their prolactinomas and not all patients with prolactinomas, which may explain some of the differences in results from previous studies, through the introduction of selection bias. As the patients at our tertiary referral institution were more often initially treated at outside centers, some of the data collected may have been subject to recall bias, including presenting symptoms, initial dopamine level at diagnosis, and length of medical therapy. In addition, four patients had previously undergone surgical treatment for their prolactinoma at other institutions; however, due to the low number, they were not included in the analysis.

\section{Conclusion}

In this retrospective series, we found that male prolactinoma patients undergoing surgical therapy differ significantly from female prolactinoma patients, with larger tumor sizes, higher frequency of suprasellar extension, and higher frequency of cavernous sinus invasion. Females were also significantly more likely to receive gross total resection during their operations and have normalization of their serum prolactin levels 2 months after surgery compared with males. We also confirmed previous findings that there are differences in presenting symptoms described between males and females, with males presenting more often with decreased libido, whereas females presented most often with menstrual irregularities and galactorrhea. Earlier diagnosis in males may improve outcomes, but this is a diagnostic challenge due to the subtle clinical presentation in males compared with females.

Note

A portion of the data presented in this article was presented at the North American Skull Base Society meeting on March 3-5, 2017, in New Orleans, LA.

\section{Conflict of Interest}

There is no financial disclosure or conflict of interest to disclose for any of the authors. 


\section{References}

1 Tampourlou M, Trifanescu R, Paluzzi A, Ahmed SK, Karavitaki N. THERAPY OF ENDOCRINE DISEASE: surgery in microprolactinomas: effectiveness and risks based on contemporary literature. Eur J Endocrinol 2016;175(03):R89-R96

2 Melmed S, Casanueva FF, Hoffman AR, et al; Endocrine Society. Diagnosis and treatment of hyperprolactinemia: an Endocrine Society clinical practice guideline. J Clin Endocrinol Metab 2011; 96(02):273-288

3 Webster J, Piscitelli G, Polli A, Ferrari CI, Ismail I, Scanlon MF; Cabergoline Comparative Study Group. A comparison of cabergoline and bromocriptine in the treatment of hyperprolactinemic amenorrhea. N Engl J Med 1994;331(14):904-909

4 Colao A. Pituitary tumours: the prolactinoma. Best Pract Res Clin Endocrinol Metab 2009;23(05):575-596

5 Cho DY, Liau WR. Comparison of endonasal endoscopic surgery and sublabial microsurgery for prolactinomas. Surg Neurol 2002; 58(06):371-375, discussion 375-376

6 Dehdashti AR, Ganna A, Karabatsou K, Gentili F. Pure endoscopic endonasal approach for pituitary adenomas: early surgical results in 200 patients and comparison with previous microsurgical series. Neurosurgery 2008;62(05):1006-1015, discussion 10151017

7 Akin S, Isikay I, Soylemezoglu F, Yucel T, Gurlek A, Berker M. Reasons and results of endoscopic surgery for prolactinomas: 142 surgical cases. Acta Neurochir (Wien) 2016;158(05):933-942

8 Dallapiazza RF, Jane JA Jr. Outcomes of endoscopic transsphenoidal pituitary surgery. Endocrinol Metab Clin North Am 2015;44 (01):105-115

9 Molitch ME. Diagnosis and treatment of pituitary adenomas: a review. JAMA 2017;317(05):516-524

10 Mindermann T, Wilson CB. Age-related and gender-related occurrence of pituitary adenomas. Clin Endocrinol (Oxf) 1994;41(03): 359-364

11 Ramot Y, Rapoport MJ, Hagag P, Wysenbeek AJ. A study of the clinical differences between women and men with hyperprolactinemia. Gynecol Endocrinol 1996;10(06):397-400

12 Delgrange E, Trouillas J, Maiter D, Donckier J, Tourniaire J. Sexrelated difference in the growth of prolactinomas: a clinical and proliferation marker study. J Clin Endocrinol Metab 1997;82(07): 2102-2107

13 Delgrange E, Vasiljevic A, Wierinckx A, et al. Expression of estrogen receptor alpha is associated with prolactin pituitary tumor prognosis and supports the sex-related difference in tumor growth. Eur J Endocrinol 2015;172(06):791-801

14 Fainstein Day P, Glerean M, Lovazzano S, et al. Gender differences in macroprolactinomas: study of clinical features, outcome of patients and ki-67 expression in tumor tissue. Front Horm Res 2010;38:50-58
15 Colao A, Sarno AD, Cappabianca P, et al. Gender differences in the prevalence, clinical features and response to cabergoline in hyperprolactinemia. Eur J Endocrinol 2003;148(03):325-331

16 Colao A, Lombardi G. Growth-hormone and prolactin excess. Lancet 1998;352(9138):1455-1461

17 Verhelst J, Abs R, Maiter D, et al. Cabergoline in the treatment of hyperprolactinemia: a study in 455 patients. J Clin Endocrinol Metab 1999;84(07):2518-2522

18 Ciccarelli A, Guerra E, De Rosa M, et al. PRL secreting adenomas in male patients. Pituitary 2005;8(01):39-42

19 Burrow GN, Wortzman G, Rewcastle NB, Holgate RC, Kovacs K. Microadenomas of the pituitary and abnormal sellar tomograms in an unselected autopsy series. N Engl J Med 1981;304(03):156-158

20 Gooren LJ, Assies J, Asscheman H, de Slegte R, van Kessel H. Estrogen-induced prolactinoma in a man.J Clin Endocrinol Metab 1988;66(02):444-446

21 Kovacs K, Stefaneanu L, Ezzat S, Smyth HS. Prolactin-producing pituitary adenoma in a male-to-female transsexual patient with protracted estrogen administration. A morphologic study. Arch Pathol Lab Med 1994;118(05):562-565

22 Serri O, Noiseux D, Robert F, Hardy J. Lactotroph hyperplasia in an estrogen treated male-to-female transsexual patient. J Clin Endocrinol Metab 1996;81(09):3177-3179

23 García-Malpartida K, Martín-Gorgojo A, Rocha M, Gómez-Balaguer M, Hernández-Mijares A. Prolactinoma induced by estrogen and cyproterone acetate in a male-to-female transsexual. Fertil Steril 2010;94(03):1097.e13-1097.e15

24 Delgrange E, Sassolas G, Perrin G, Jan M, Trouillas J. Clinical and histological correlations in prolactinomas, with special reference to bromocriptine resistance. Acta Neurochir (Wien) 2005;147 (07):751-757, discussion 757-758

25 Robert F. Morphology of human prolactinomas: a study on invasive prolactin-secreting adenomas. In: Tolis G, ed. Prolactin and Prolactinomas. New York: Raven; 1983:339-349

26 Faraoni EY, Camilletti MA, Abeledo-Machado A, et al. Sex differences in the development of prolactinoma in mice overexpressing hCGß: role of TGFß1. J Endocrinol 2017;232(03):535-546

27 Arasho BD, Schaller B, Sandu N, Zenebe G. Gender-related differences in pituitary adenomas. Exp Clin Endocrinol Diabetes 2009; 117(10):567-572

28 Oh MC, Aghi MK. Dopamine agonist-resistant prolactinomas. J Neurosurg 2011;114(05):1369-1379

29 Bhasin S, Cunningham GR, Hayes FJ, et al; Task Force, Endocrine Society. Testosterone therapy in men with androgen deficiency syndromes: an Endocrine Society clinical practice guideline. J Clin Endocrinol Metab 2010;95(06):2536-2559

30 Leonard MP, Nickel CJ, Morales A. Hyperprolactinemia and impotence: why, when and how to investigate. J Urol 1989;142(04): 992-994 